\title{
A Method for Registering Diffusion Weighted Magnetic Resonance Images
}

\author{
Xiaodong Tao and James V. Miller \\ GE Research, Niskayuna, New York, USA
}

\begin{abstract}
Diffusion weighted magnetic resonance (DWMR or DW) imaging is a fast evolving technique to investigate the connectivity of brain white matter by measuring the self-diffusion of the water molecules in the tissue. Registration is a key step in group analysis of the DW images that may lead to understanding of functional and structural variability of the normal brain, understanding disease process, and improving neurosurgical planning. In this paper, we present a new method for registering DW images. The method works directly on the diffusion weighted images without using tensor reconstruction, fiber tracking, and fiber clustering. Therefore, the performance of the method does not rely on the accuracy and robustness of these steps. Moreover, since all the information in the original diffusion weighted images is used for registration, the results of the method is robust to imaging noise. We demonstrate the method on intra-subject registration with an affine transform using DW images acquired on the same scanner with the same imaging protocol. Extension to deformable registration for images acquired on different scanners and/or with different imaging protocols is also discussed.
\end{abstract}

This work is part of the National Alliance for Medical Image Computing, funded by the National Institutes of Health through the NIH Roadmap for Medical Research, Grant U54 EB005149. Information on the National Centers for Biomedical Computing can be obtained from http://nihroadmap.nih.gov/bioinformatics.

\section{Introduction}

Diffusion is a process caused by Brownian motion of molecules. From the microscopic viewpoint and within a small enough time period, the motion is completely random, governed by thermal dynamics. When observed at a relatively large scale, the diffusion is often limited by the structure of the substance in which the molecules reside. By investigating the diffusion properties of molecules in a sample, one can infer the structure of the sample. Diffusion weighted magnetic resonance imaging is an emerging modality that is able to measure the diffusivity in a sample [1|2]. Its use in the area of medical imaging has drawn increasing interest over the past decade. DW imaging is a powerful tool for neurologists in mapping the brain white matter fibers and has a great potential to elicit the functional organization, development, aging, and disease process of the brain [3|4 56]. DW imaging also plays an important role for neurosurgical planning [7].

Group analysis is essential for understanding normal variability and detecting abnormalities. It involves transforming images to a canonical coordinate frame, normalizing gross inter-subject morphological differences [8]. In the general framework of image 


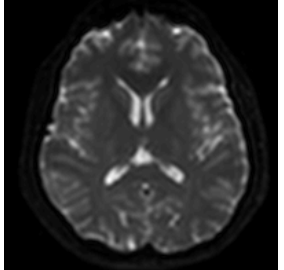

(a)

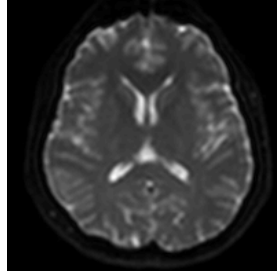

(b)

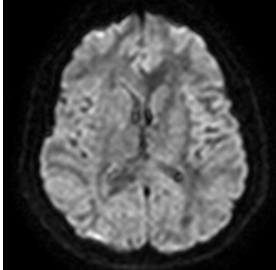

(c)

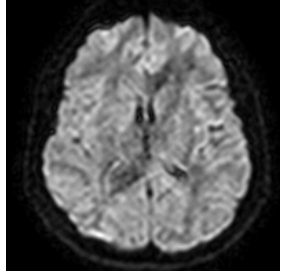

(d)

Fig. 1. T2-weighted images [(a) and (b)] and DW images [(c) and (d)] of the same subject (registered and interpolated to the same axial slice) from two different sessions. Although (a) and (b) show good alignment, (c) and (d) differ due to DW intensity dependency on patient orientation.

registration, a transformation is sought to maximize the similarity between the transformed moving image and the fixed image. One premise is that both images contain the same information that is independent of the transformation. This premise, however, is not true for DW images whose intensity values also depend on the patient orientation, and therefore on the transformation (Fig.11). The dependence of the DW measurements on patient orientation makes DW image registration fundamentally different from conventional registration of T1 and T2 weighted images.

Previous approaches for registering DW images usually decompose the DW images into an orientation independent part (e.g., a fractional anisotropy (FA) map) and an orientation dependent part (e.g., a diffusion tensor map or a principal direction map). The orientation independent parts are registered and the orientation dependent parts are reoriented by the transformation from the orientation independent part [9 10 $11 \mid 12$ 13]. Note, the orientation independent parts are usually derived from a tensor estimation or are based on an extra $\mathrm{T} 1$ acquisition. Therefore, the accuracy of DW image registration depends on these steps.

In this paper, we develop a unified framework for DW image registration, which works directly on the DW images without requiring tensor reconstruction, or co-registration of DW images with corresponding T1 weighted images. We demonstrate the new algorithm on intra-subject registration using affine transforms. We also compare the proposed algorithm with an algorithm based on reorientation of principal diffusion directions. Finally, we discuss how this new registration framework can be extended into more general deformable registration.

\section{Background}

In organized tissues, such as brain white matter, water molecules undergo anisotropic self diffusion, which is often modeled by the self-correlation function $P(\mathbf{r} \mid \mathbf{r}+\mathbf{R}, t)$ (using the nomenclature from [1]). The self-correlation function can be thought of as the probability that a molecule initially at $\mathbf{r}$ will move to location $\mathbf{r}+\mathbf{R}$ after a time of $t$. The average propagator is defined as:

$$
\bar{P}(\mathbf{R}, t)=\int P(\mathbf{r} \mid \mathbf{r}+\mathbf{R}, t) \rho(\mathbf{r}) d \mathbf{r}
$$


where $\rho(\mathbf{r})$ is the proton density. Under certain assumptions (e.g., narrow gradient pulse), the average propagator is related to the measured MR diffusion signal by the Fourier relationship:

$$
E(\mathbf{q})=\int \bar{P}(\mathbf{R}, t) e^{i 2 \pi \mathbf{q} \cdot \mathbf{R}} d \mathbf{R},
$$

where $\mathbf{q}=(2 \pi)^{-1} \gamma \delta \mathbf{g}, \gamma$ is the gyromagnetic ratio for the protons, $\delta$ is the diffusion gradient duration, and $\mathbf{g}$ is a diffusion gradient vector. The above equation has been normalized such that $E(\mathbf{q})=1$ for $\mathbf{q}=\mathbf{0}$. In practice, one or more T2-Weighted baseline images are acquired to properly normalize the diffusion weighted images. The MR signal, $E(\mathbf{q})$, is used to reconstruct the average propagator, $\bar{P}(\mathbf{R}, t)$, based on a model for diffusion (e. g., anisotropic Gaussian diffusion). When studying orientational structure of tissue, it is often sufficient to limit the diffusion gradient vectors to be unit vectors. Under these conditions, $E(\mathbf{q})$ in Eq. (2) can be viewed as samples drawn from a function defined on the unit sphere. In the following, we will denote a DW image as $I(\mathbf{r}, \mathbf{g})$, with $\mathbf{r} \in \Re^{3}$ and $\|\mathbf{g}\|=1$.
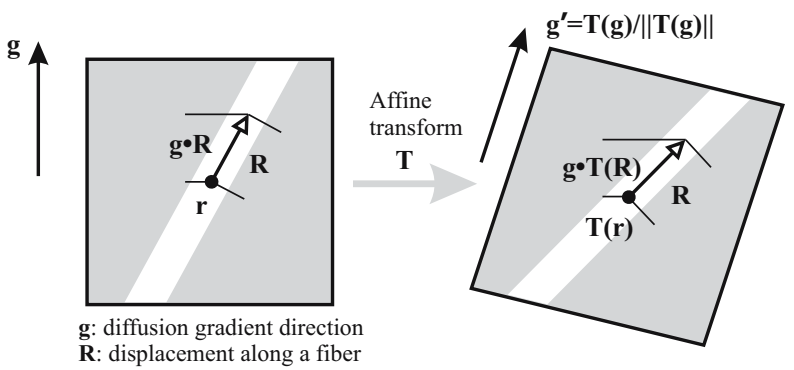

Fig. 2. A voxel is transformed by an affine transform $\mathbf{T}$. Under such a transform, the projection of the displacement vector $\mathbf{R}$ on the diffusion gradient direction changes from $\mathbf{g} \cdot \mathbf{R}$ to $\mathbf{g} \cdot \mathbf{T}(\mathbf{R})$.

From Eq. (2), we can see that the diffusion MR signal depends on the relative position of the tissue sample and the diffusion gradient vector $\mathbf{g}$. Usually $\mathbf{g}$ is defined in the world coordinate system and is fixed for a given imaging protocol. Therefore, $I(\mathbf{r}, \mathbf{g})$ depends on the patient orientation with respect to the scanner. For a constant $\mathbf{g}$ and a given anatomic location, the MR signal, $I(\mathbf{r}, \mathbf{g})$, varies with the orientation of $\mathbf{r}$ with respect to $g$. This is illustrated in Fig. 2. The orientation dependency of the DW images differs from conventional T1-weighted and T2-weighted images and makes it difficult to compare DW images directly. In Section 3.1, we describe an angular interpolation algorithm that enables a direct comparison of DW images. The angular interpolation algorithm is the basis of our registration method for DW images.

\section{Registration Framework}

Many image registration algorithms exist in the literature. The majority can be formulated as a general optimization problem. Given two images in 3-dimensional space, a 
fixed image $I_{1}(\mathbf{r})$ and a moving image $I_{2}(\mathbf{r}), \mathbf{r} \in \Re^{3}$, a transformation $\mathbf{T}$ is sought to minimize a cost function:

$$
\mathcal{E}[\mathbf{T}]=\mathcal{D}\left[I_{1}(\mathbf{r}), \mathbf{T} \circ I_{2}(\mathbf{r})\right],
$$

where $\mathcal{D}$ is a measure of difference between two images. $\mathcal{D}$ can be the mean squared error between image intensities, distances between corresponding pairs of landmarks, or information theoretic measures such as mutual information. Generally speaking, there are four major components for a registration task [14]: a metric [D in Eq. (3)] that defines the difference measure between images; a transformation $\mathbf{T}$ that defines the class of transformation applied to the moving image, which may be linear (e. g., affine transform) or deformable (e. g., B-Spline deformable transform); an optimizer that searches the parameter space of the transformation; and an interpolation method that interpolates image intensities in the transformed moving image.

\subsection{Angular Interpolation of Diffusion Weighted Images}

As shown in Fig. 1, registered and resampled T2-weighted images can accurately align the anatomy, yet the resampled DW images can be quite different due to the DW MR signal's dependency on patient orientation. To address the orientation dependency, we need to transform diffusion weighting gradient directions in accordance with the transform that aligns the patient anatomy. We will describe an angular interpolation algorithm in this section.

The MR diffusion signals $E(\mathbf{g})$ is a function defined on the unit sphere. The measurements are samples of this function in the given diffusion gradient directions. Given $N$ diffusion gradient directions $\mathbf{g}_{i}$, with $\left\|\mathbf{g}_{i}\right\|=1$, the intensity of DW image with a diffusion gradient direction $\mathbf{k}$ at a spatial location $\mathbf{r}$ is

$$
I(\mathbf{r}, \mathbf{k})=\left(\sum_{i=1}^{N} w_{i} I\left(\mathbf{r}, \mathbf{g}_{i}\right)\right) /\left(\sum_{i=1}^{N} w_{i}\right),
$$

where $w_{i}=e^{-d^{2} / 2 \sigma^{2}}$, with $d=\cos ^{-1}\left|\mathbf{k} \cdot \mathbf{g}_{i}\right|$ being the geodesic distance on the sphere between the gradient direction and the interpolated direction, and $\sigma$ being a parameter that controls the smoothness of the interpolation operator. Other weighting schemes based on angular difference can also be used. By taking the absolute value of the dot product of $\mathbf{k}$ and $\mathbf{g}_{i}$, we assume the image $I(\mathbf{r}, \mathbf{g})$ is symmetric in $\mathbf{g}$, i.e., $I(\mathbf{r}, \mathbf{g})=I(\mathbf{r},-\mathbf{g})$. Using the interpolation of Eq. (4), we can estimate a diffusion MR measurement in any direction, given that the underlying orientational structure of tissue is fairly smooth. We note that when $\mathbf{k}=\mathbf{g}_{i}$ for some $i$, Eq. (4) becomes a smoothing operation on the DW images, which can serve as a pre-processing step to reduce noise. This angular smoothing operation uses information at a given voxel from all diffusion gradient directions. Moreover, it does not spatially average across voxels.

\subsection{Resampling DW Images Using Angular Interpolation}

The angular interpolation algorithm can be used to transform and resample DW images to have consistent diffusion gradient directions. Given an affine transform $\mathbf{T}$, we can 
represent it by a $3 \times 3$ transformation matrix, $M$, and a translation vector, $\mathbf{t}$. Using this representation, a point $\mathbf{r}$ and a diffusion gradient vector $\mathbf{g}$ are transformed into (see Fig. 2]

$$
\mathbf{r}^{\prime}=M \cdot \mathbf{r}+\mathbf{t} \text {, and } \mathbf{g}^{\prime}=M \cdot \mathbf{g} /\|M \cdot \mathbf{g}\| .
$$

The vector $\mathbf{g}^{\prime}$ is normalized to have unit length because we only care about its direction. Therefore, an affine transform $\mathbf{T}$ will transform a DW image $I(\mathbf{r}, \mathbf{g})$ into

$$
I^{\prime}(\mathbf{r}, \mathbf{g})=I\left(M^{-1}(\mathbf{r}-\mathbf{t}), \frac{M^{-1} \mathbf{g}}{\left\|M^{-1} \mathbf{g}\right\|}\right)
$$

using Eqs. (4). Note if we can register T2 weighted image, we could use the resulting transformation to reorient the DW images using the above equation.

\subsection{Registration of Diffusion Weighted Images}

Although it is possible to use T2 weighted images to obtain a transform that aligns two DW image sets, much more information is contained in diffusion weighted images. By using the complete information, we will get more accurate and robust results. We now describe a method to directly register DW images using the angular interpolation. For the purpose of discussion, we will use a mean squared error (MSE) and an affine transform in the following. Extension to other image metric is straightforward. Extension to deformable registration can be achieved by approximating a deformable transform with locally affine transforms.

Given two sets of DW images, $\mathbf{I}_{1}(\mathbf{r})$ and $\mathbf{I}_{2}(\mathbf{r}), \mathbf{r} \in \Re^{3}$, that consist of volumetric images in $M$ and $N$ diffusion gradient directions, respectively. The volumetric images are denoted as

$$
I_{1}\left(\mathbf{r}, \mathbf{g}_{i}^{(1)}\right) \text { and } I_{2}\left(\mathbf{r}, \mathbf{g}_{j}^{(2)}\right), i=1, \cdots, M, j=1, \cdots, N .
$$

Note that $\mathbf{I}_{1}(\mathbf{r})$ and $\mathbf{I}_{2}(\mathbf{r})$ can have different gradient direction. Therefore, the proposed method is able to register DW images acquired with different protocols. The intensity of the DW images are properly normalized and bias corrected. So, mean square error can be used as an image similarity measure. The objective of registration is to find the affine transformation $\mathbf{T}$ (composed of a transformation matrix $M$ and a translation vector $\mathbf{t}$ ) that minimizes the MSE between $\mathbf{I}_{1}(\mathbf{r})$ and transformed $\mathbf{I}_{2}(\mathbf{r})$, which is given by [see Eq. (6)]

$$
\mathcal{E}(M, \mathbf{t})=\sum_{i=1}^{M} \int_{\mathbf{r}}\left[I_{1}\left(\mathbf{r}, \mathbf{g}_{i}^{(1)}\right)-I_{2}\left(M^{-1}(\mathbf{r}-\mathbf{t}), \frac{M^{-1} \mathbf{g}_{i}^{(1)}}{\left\|M^{-1} \mathbf{g}_{i}^{(1)}\right\|}\right)\right]^{2} d \mathbf{r} .
$$

The transform, $\mathbf{T}$, not only maps a point $\mathbf{r}$ in the fixed image domain to an anatomically corresponding point $\mathbf{r}^{\prime}$ in the moving image domain, but also interpolates the DW image intensity at $\mathbf{r}^{\prime}$ in the properly transformed diffusion gradient direction using measurements at that location from all directions. Effectively, the spatial transformation "moves" the patient so that the same anatomic feature is at the same location with 
respect to the scanner, and the angular interpolation "rotates" $\mathbf{g}_{i}$ so that a consistent relationship between $\mathbf{g}_{i}$ and tissue orientation is achieved. We use a gradient descent method to solve Eq. (7). Other optimization algorithms can also be used.

The registration algorithm is implemented using the Insight Segmentation and Registration Toolkit (ITK) [15], which provides rich sets of transformations, image metrics, optimizers, and interpolation algorithms that can be customized for different applications. In our implementation, we use an affine transform, a mean square error metric, and a gradient descent optimizer. The transformed images are interpolated using a linear interpolation algorithm. The new angular interpolation algorithm is used to compute MSE between DW images.

\section{Experimental Results}

Two sets of DW images were acquired for one subject with the same imaging protocol. Each set of DW images has 5 baseline T2 weighted images and 30 diffusion weighted images. The image resolution is $0.9375 \times 0.9375 \times 2.5 \mathrm{~mm}^{3}$. The two sets of images are denoted as $\mathbf{I}_{1}$ and $\mathbf{I}_{2}$ for session one and session two. $\mathbf{I}_{1}$ is used as the fixed image. $\mathbf{I}_{2}$ is registered to $\mathbf{I}_{1}$, resulting in a transform $\mathbf{T}$. Based on our experiments, we fixed $\sigma$ to be the average of the geodesic distances between neighboring directions in all the experiments.

Fig. 3 shows the axial slices of a DW image with same diffusion weighting gradient from $\mathbf{I}_{1}$, transformed $\mathbf{I}_{2}$, and transformed $\mathbf{I}_{2}$ with angular interpolation. From the image, we can see that angular interpolation introduces blurring, but the image with angular interpolation does reveal same tissue orientation at same place. The regions highlighted by the boxes are zoomed in for better visualization in each images.

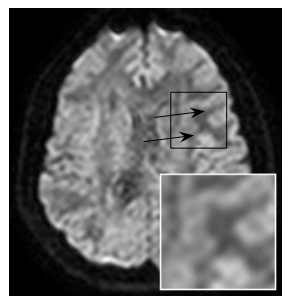

(a)

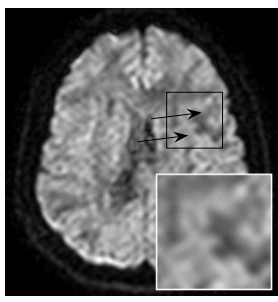

(b)

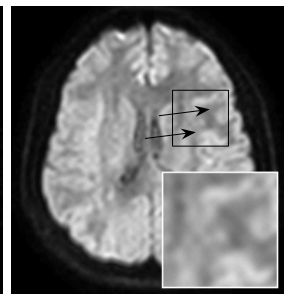

(c)

Fig. 3. An axial slice from $\mathbf{I}_{1}$ (a); same slice from transformed $\mathbf{I}_{2}$ (b); and transformed $\mathbf{I}_{2}$ with angular interpolation (c). The images have same diffusion weighting gradient w.r.t. the scanner.

Fig. 4 (a) shows the fractional anisotropy map of an axial slice of $\mathbf{I}_{1}$; Fig. 4 (b) shows the same slice of the transformed FA map of $\mathbf{I}_{2}$; and Fig. 4 (c) shows the FA map computed from the registered $\mathbf{I}_{2}$. The same structures can be seen in all the images. Because of the extra step of angular interpolation, the FA map showed in Fig. 4 (c) has less contrast.

In Fig. 5, tractography results are overlaid on a coronal slice of FA maps. We used a streamline based tractography algorithm similar to [16]. For all tractography, the same 


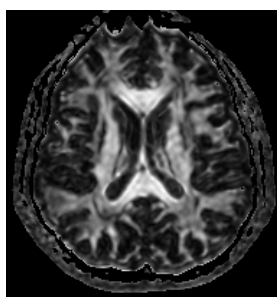

(a)

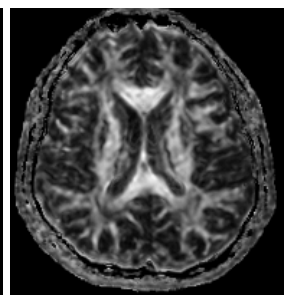

(b)

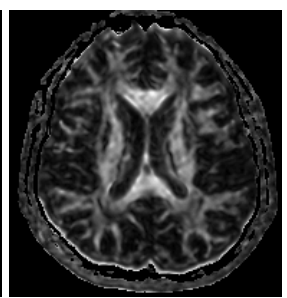

(c)

Fig. 4. An axial slice of the FA map of $\mathbf{I}_{1}$ (a), the transformed FA map of $\mathbf{I}_{2}$ (b), and the FA map of the registered $\mathbf{I}_{2}$ (c)

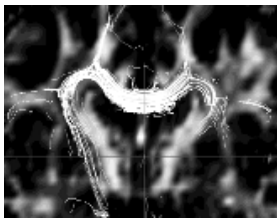

(a)

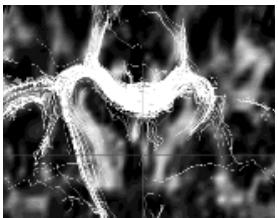

(b)

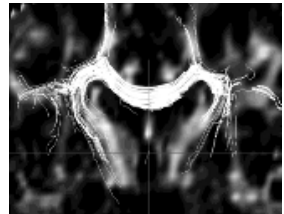

(c)

Fig. 5. Fiber tracts of the fixed DW images (a), registration using only T2 weighted images (b), and registration using entire DW image set (c)

set of parameters are used; the seed points are located near mid-sagittal plane and have the same coordinates. Fig. 5 (a) shows fiber tracts obtained from the fixed image $\mathbf{I}_{1}$; Fig.5(b) shows fiber tracts obtained by reorienting principle directions using a method similar to [9]; and Fig. 5] (c) shows fiber tracts obtained from the registered $\mathbf{I}_{2}$ using the proposed method. The figures clearly show that fiber tracts in (a) and (c) reveal the same white matter structures. In (b) the fiber tracts deviate from the underlying WM structures, although the FA map look similar to the ones in (a) and (c). The deviation is primarily due to the inconsistency between the registration and tensor reorientation.

\section{Summary and Discussion}

In this paper, we presented a novel method for registering diffusion weighted MR images. The method works directly on the DW images without using tensor reconstruction or referencing co-registered T1 weighted images. We developed an angular interpolation algorithm that allows us to estimate DW image intensity at a given location in any direction from the acquired DW images. This interpolation algorithm effectively rotates the patient into a consistent orientation so that direct comparison of DW images with same diffusion gradient direction is meaningful. We showed preliminary results of registering DW images of the same subject acquired in two imaging sessions. Because of the smoothing effect of the angular interpolation, the DW images after interpolation is smoother and the FA of the registered images is smaller. The principal diffusion directions are well preserved, indicated by fiber tracking results.

There are several advantages of the proposed method. Firstly, by using all the information in the original DW images, the registration is robust. The cost function [Eq. (7)] 
depends on DW images in all diffusion directions. It is minimized only when all images are consistently aligned. Secondly, the method does not depend on tensor reconstruction or reference to co-registered T1 weighted images. Therefore, its performance does not rely on the accuracy and robustness of these steps. And thirdly, the method fits well in the unified registration framework without requiring an extra step to reorient tensors or principal diffusion directions.

Validation is a difficult but important topic for the future. More studies will be done to compare the proposed method with existing ones. In this paper, we used an affine transform to register DW images of same subject acquired in two sessions. Extending the algorithm to non-rigid registration of DW images of different subjects will be very interesting, and have broader applications. Future work also includes applying the method to more datasets and studying variability of diffusion properties of the brain white matter.

\section{Acknowledgment}

The authors would like to thank Dr. Susumu Mori at Department of Radiology Johns Hopkins University, School of Medicine for providing the datasets used in this paper.

\section{References}

1. P. T. Callaghan, "Principles of Nuclear Magnetic Resonance Microscopy", Oxford: Oxford University Press, 1993.

2. P. Basser, J. Mattiello and D. LeBihan, "Estimation of the Effective Self-Diffusion Tensor from teh NMR Spin Echo", Journal of Magnetic Resonance, B, 103:247-254, 1994.

3. C. Pierpaoli, P. Jezzard, P. J. Basser, A. Barnett and G. Di Chiro, "Diffusion Tensor MR Imaging of the Human Brain", Radiology, 201: 637-648, 1996.

4. D. S. Tuch, T. G. Reese, M. R. Wiegell and V. J. Wedeen, "Diffusion MRI of Complex Neural Architecture", Neuron, 40:885-895, 2003.

5. P.S. Huppi, S.E. Maier, S. Peled, G.P. Zientara, P.D. Barnes, F.A. Jolesz and J.J. Volpe, "Microstructural development of human newborn cerebral white matter assessed in vivo by diffusion tensor magnetic resonance imaging", Pediatric Research, 44:584-590, 1998.

6. J. Foong, M. Maier, C. A. Clark, G. J. Barker, D. H. Miller and M. A. Ron, "Neuropathological abnormalities of the corpus callosum in schizophrenia: a diffusion tensor imaging study", J Neurol Neurosurg Psychiatry, 68:242-244, 2000.

7. C. A. Clark, T. R. Barrick, M. M. Murphy and B. A. Bell, "White matter fiber tracking in patients with space-occupying lesions of the brain: a new technique for neurosurgical planning?", Neuroimage, 20: 1601-1608, 2003.

8. J. B. A. Maintz and M. A. Viergever, "A survey of medical image registration", Medical Image Analysis, 2:1-36, 1998.

9. D. C. Alexander, C. Pierpaoli, P. J. Basser and J. C. Gee, "Spatial Transformations of Diffusion Tensor Magnetic Resonance Images", IEEE Trans. Med. Imag., 1131-39, 2001.

10. D. K. Jones, L. D. Griffin, D. C. Alexander, M. Catani, M. A. Horsfield, R. Howard and S. C. R. Williams, "Spatial Normalization and Averaging of Diffusion Tensor MRI Data Sets", NeuroImage, 17:592-617, 2002.

11. D. Xu, S. Mori, D. Shen, P.C.M. van Zijl and C. Davatzikos, "Spatial Normalization of Diffusion Tensor Fields", Magnetic Resonance in Medicine, 50:175-182, 2003. 
12. H.-J. Park, M. Kubicki, M.E. Shenton, A. Guimond, R.W. McCarley, S.E. Maier, R. Kikinis, F.A. Jolesz and C.-F. Westinb, "Spatial normalization of diffusion tensor MRI using multiple channels", Neuroimage, 20:1995-2009, 2003.

13. O. Ciccarelli, A.T. Toosy, G.J.M. Parker , C.A.M. Wheeler-Kingshott, G.J. Barker, D.H. Miller and A.J. Thompsona, "Diffusion tractography based group mapping of major whitematter pathways in the human brain", Neuroimage, 19:1545-1555, 2003.

14. T. Yoo (editor), "Insight into Images: Principles and Practice for Segmentation, Registration, and Image Analysis", A. K. Peters, Ltd., 2004.

15. L. Ibánẽz, W. Schroeder, L. Ng and J. Cates, "The ITK Software Guide: The Insight Segmentation and Registration Toolkit", Kitware Inc., 2003.

16. D. Weinstein, G. Kindlmann and E. C. Lundberg, "Tensorlines: advection diffusion based propagation through diffusion tensor fields", In Proceedings, IEEE Visualization, San Francisco, CA, 1999; p. 249-253. 